\title{
Research on evolution region of self-similar pulses in a dispersion-decreasing fiber
}

\author{
QiaoFen Zhang, Huaizhong Li, Liming Wu, Jian Gao, Guitang Wang, Yaohua deng
}

1 Key Laboratory of Precision Microelectronic Manufacturing Technology \& Equipment of Ministry of Education of Guangdong University of Technology, Guangzhou, 510006, China

2 School of Engineering and Build Environment, Gold Coast campus, Griffith University, QLD 4222, Australia

Telephones: $+86-20-87796616$

Fax: $+86-20-87796616$

E-mail: zhqf@gdut.edu.cn

Abstract:

The relationship between the self-similar propagation region for single pulse and oscillation region for a pair of self-similar pulses are first investigated in our paper. By introducing self-similar coefficient $F$ and RMS width ratio $K$, we find that self-similar propagation region starts from $z=1.8 L_{D}$ to $z=18 L_{D}$ while $F \leq 10 \%$. The optimum output of self-similar pulse is also achieved when $F$ and $K$ reach a minimum value simultaneously at $z=3.5 L_{D}$. The sinusoidal fit oscillation region of self-similar pulse pair ranges from $5 / 8 L_{D}$ to $2 L_{D}$ while $F$ varies from $40.27 \%$ to $7.99 \%$, and the dark soliton fit oscillation region ranges from $2 L_{D}$ to $6 L_{D}$ while $F$ varies from $7.99 \%$ to $5.32 \%$, indicating that the sinusoidal fit oscillation region almost occurs before the pulses enter the self-similar propagation region and the dark soliton fit oscillation region occurs within the self-similar pulse propagation region. Furthermore, the oscillation characteristics of interacting pulses are also studied numerically by using split-step Fourier method. The results are beneficial in Dense Wavelength Division Multiplexing transmission system which is in heavy demands of light source in wide-range wavelength.

Keywords: ND-DDF; nonlinear Schrödinger equation; self-similar pulses; self-similar propagation region; sinusoidal fit oscillation region; asymptotic dark soliton oscillation region

Abbreviations: ND-DDF: dispersion-decreasing optical fiber with normal group-velocity dispersion 


\section{Introduction}

Self-similar pulse is a more general class of shape-preserving waves, which maintains its parabolic shape of the envelope but the parameters such as amplitude, width, and chirp evolve with propagation inside nonlinear media (Barenblatt, G.I. 1996). The self-similar pulse does not suffer from the deadly effects of optical wave breaking (Desaix, M. 1993). These attractive features lead parabolic pulse to a wide-range practical significance, such as in the generation of high-power femtosecond lasers (Ruehl et al. 2006), spectral broadening and supercontinuum generation (Tamura et al. 2000), multi-wavelength source (Ozeki et al. 2004), spectrally sliced pulse source generation (Wang et al. 2016) and pulse reshaping (Boscolo et al. 2008). Previous researches concerned more with the generation and propagation properties of single self-similar pulse in a variety of fibers (Hirooka et al. 2004; Kibler et al. 2006; Wabnitz et al. 2008; Ghosh et al. 2009; Bale et al. 2010; Ghosh et al.2017). Meanwhile, more and more studies also focus on the interaction and propagation of a pair of parabolic pulses, such as interaction between parabolic pulses based on fiber amplifier (He et al. 2014; Finot et al. 2005; Finot et al. 2006), dispersion-managed fiber (Liu et al. 2011; Ponomarenko et al. 2008), nonlinear waveguide (Loomba et al. 2014; Kumar et al. 2015), highly nonlinear fibers (Ghosh et al. 2015; Krishna et al. 2018) and ND-DDF (Zhang et al. 2011). However, to our knowledge, little attention has been given to the relationship between the oscillation region of a pair of self-similar pulses and the self-similar pulse propagation region, within which the parabolic pulse can keep their shape without distortion and 
oscillations, in ND-DDF. In this paper, we first investigate the relationship among the self-similar propagation region for single pulse, sinusoidal fit oscillation region and asymptotic dark soliton oscillation region for a pair of self-similar pulses. By introducing self-similar coefficient $F$ and RMS width ratio $K$, we demonstrate that self-similar propagation is only effective in a limited region and the optimum output of self-similar pulse is also achieved. By considering self-similar coefficient $F$, the relationship between the oscillation region and the self-similar propagation region are analyzed. Meanwhile, the oscillation characteristics of interacting pulses are also studied numerically by using split-step Fourier method.

\section{Self-similar propagation region of single pulse evolving in ND-DDF}

The propagation of optical pulses in a ND-DDF is modeled by an NLS (nonlinear

Schrödinger) equation of the form

$$
i \frac{\partial A}{\partial z}-\frac{\beta_{2}}{2} D(z) \frac{\partial^{2} A}{\partial T^{2}}+\gamma|A|^{2} A=0
$$

where $z$ is the fiber length, $T$ is the time measured in a frame of reference moving with the pulse at the group velocity $v_{g}\left(T=t-z / v_{g}\right)$, and $A(z, T)$ is the slowly varying amplitude of the pulse envelope, $D(z)$ represents the variation in the GVD due to dispersion tapering and is normalized such that $D(0)=1 . \beta_{2}$ and $\gamma$ are the GVD value at $z=0$ and the nonlinearity coefficient respectively. Due to the special engineering requirements and different processes, DDF can be tapered with various dispersion profile structures (2006 Silva et al. 1999; Amir et al. 1997; Zhang et al. 2019). Here we use hyperbolic dispersion tapering in a passive fiber so that the propagating pulse 
obtains the equivalent (noise-free) gain necessary for parabolic pulse generation, it means $D(z)=\frac{1}{1+g_{0} z}$. Here $g_{0}$ is a constant gain coefficient. The detailed theory parabolic asymptotic solution under this condition can be obtained from Hirooka and Masataka (2004).

To study the self-similar propagation region, we suppose that a Gaussian pulse is launched into ND-DDF, which has a FWHM (full width at half maximum) of 1.0ps and a pulse energy $E$ of 50pJ. The ND-DDF has a $\beta_{2}=1.3 p s^{2} / \mathrm{km} \gamma=4 \mathrm{~W}^{-1} \mathrm{~km}^{-1}$ and $g_{0}$ $=0.025 \mathrm{~m}^{-1}$. The single pulse evolution in the ND-DDF is showed in Fig. 1 .

The self-similar propagation region is determined in which the parabolic self-similar pulse has formed before the oscillations occur. Here we introduce self-similar coefficient $F$ and RMS width ratio $K$ to judge the effective self-similar pulse and then determine self-similar propagation region. A self-similar coefficient is to measure the degree of approximation between the numerical output pulse and the theoretical self-similar pulse, which is defined as

$$
F=\frac{\sup _{-\infty<z<\infty}\left|\int_{-\infty}^{\infty}\left(|A(z, T)|^{2}-|\hat{A}(z, T)|^{2}\right) d T\right|}{\sup _{-\infty<z<\infty} \int_{-\infty}^{\infty}|\hat{A}(z, T)|^{2} d T}
$$

where $A(z, T)$ and $\hat{A}(z, T)$ represent the numerical solution and theoretical solution of output amplitude, respectively. Since $F$ is defined as the difference of pulse energy between the numerical solution and theoretical solution, the ideal value of $F$ is zero, which means numerical output pulse is exactly equal to the theoretical self-similar pulse profile. Output pulse is more similar to parabolic profile with a smaller $F$. We perform extensive numerical simulations and find that output pulse exhibits obvious 
self-similar features when $F \leq 10 \%$, which implies complete evolution of similariton with smooth profile.

Meanwhile, the width of the pulse is more accurately described by the root-mean-square (RMS) width $\sigma$, which is defined as

$$
\sigma=\left[\left\langle T^{2}\right\rangle-\langle T\rangle^{2}\right]^{1 / 2}
$$

Where $\left\langle T^{p}\right\rangle=\frac{\int_{-\infty}^{\infty} T^{p}|A(z, T)|^{2} d T}{\int_{-\infty}^{\infty}|A(z, T)|^{2} d T}$.

To see the deviation between the simulation solution and the parabolic asymptotic solution during the pulse's propagation, we further define $K$ as the RMS width ratio,

$$
\mathrm{K}=\frac{\left|\sigma_{\mathrm{N}}-\sigma_{T}\right|}{\sigma_{T}}
$$

where $\sigma_{N}$ represents the RMS width of pulse obtained from numerical solution and $\sigma_{T}$ represents RMS width of pulse obtained from parabolic asymptotic solution, respectively. The ideal value of $K$ is zero, which means the pulse's width from the numerical simulation is exactly the same as that from theoretical solution.

The relationship between self-similar coefficient and fiber length, RMS width ratio and fiber length can be obtained from the simulation, which is shown in Fig.2 respectively.

From Fig.2 (a), self-similar coefficient $F$ begins with a very large value and reduces to $10 \%$ at $z=1.8 L_{D}$, where the incident pulse starts to represent parabolic intensity profile, indicating the pulse entering the self-similar propagation region. From the simulation, the self-similar coefficient $F$ achieves a minimum value $0.27 \%$ when $z=3.5 L_{D}=0.9707 \mathrm{~km}$, while RMS width ratio $K$ achieves a minimum value $0.56 \%$ 
exactly at the same length, which means the self-similar evolution is basically consistent with the theoretical solution, resulting in the optimum output of self-similar pulse. The result from Fig.2 also shows that the self-similar coefficient and RMS width ratio have the same evolution trend with fiber length, which is further proved that the self-similar region of the pulse can be accurately characterized by the self-similar coefficient $F$. The further simulation shows that the pulses can remain self-similar evolution up to $z=18 L_{D}$ with $F \leq 10 \%$. However, the output waveform begins to oscillate when $z$ is greater than $18 L_{D}$, which means the end of the self-similar region. Therefore, from the simulation, the self-similar propagation region starts from $z=1.8 L_{D}$ and ends at $z=18 L_{D}$.

\section{Pulses oscillation characteristics in the self-similar propagation region}

To study the socillation characteristics of pulse pair in the self-similar propagation region, we suppose that a pair of Gaussian pulses separated by a time-delay $\Delta \mathrm{T}=4.5 \mathrm{ps}$ are launched into ND-DDF, which can be expressed in the follow equation.

$$
A(0, T)=\sqrt{P_{0}} \exp \left[-\frac{(T+\Delta T / 2)^{2}}{2 T_{0}^{2}}\right]+\sqrt{P_{0}} \exp \left[-\frac{(T-\Delta T / 2)^{2}}{2 T_{0}^{2}}\right]
$$

The two pulses evolution in the ND-DDF is showed in Fig.3.

Due to the linearly chirped parabolic pulses, the frequency difference between the overlapping falling and raising edges of the pulses induces a beating in the resultant signal. Zhang and Gao (2011) already showed that the pulses' overlap region oscillates with a sinusoidal fit at the beginning of their overlap and then further evolves into a train of asymptotic dark solitons. Here we further study the relationship 
between the oscillation regions and self-similar propagation region.

From Fig.3, the sinusoidal fit oscillating only generates within a short distance, and then converts to asymptotic dark solitons oscillating which continues for a long distance. Table 1 shows the relationship between the oscillation period and fiber length in the sinusoidal fit oscillation region and the asymptotic dark solitons oscillation region respectively. The oscillation period sample is taken every $1 / 8 L_{D}$ during the sinusoidal fit oscillation region, while the oscillation period sample is taken every $1 / 2 L_{D}$ as the change of oscillation period slows down in the asymptotic dark solitons oscillation region.

Fig.4 shows the relationship between the oscillation frequency and fiber length. The first-order fitting curves in sinusoidal fit oscillation region and asymptotic dark solitons oscillation region are illustrated. The fitting function in sinusoidal fit oscillation region can be written as

$$
f(z)=-13.8 z+12.1, \quad \frac{5}{8} L_{D} \leq z \leq 2 L_{D}
$$

While the fitting function in asymptotic dark solitons oscillation region is given by

$$
f(z)=-2 z+5.6, \quad 2 L_{D} \leq z \leq 6 L_{D}
$$

The second-order fitting curve in the entire oscillation region is also defined as

$$
f(z)=5.5 z^{2}-14.3 z+11.6, \quad \frac{5}{8} L_{D} \leq z \leq 6 L_{D}
$$

The pulses evolution of the overlap region is mainly divided into three parts, namely, sinusoidal fit oscillation, asymptotic dark solitons oscillation and irregular oscillation. As shown in Fig.4, the decrease rate of the oscillation frequency in the sinusoidal fit oscillation region is much higher than that in the asymptotic dark solitons oscillation 
region. As the pulses propagate from $z=5 / 8 L_{D}$ to $z=2 L_{D}$, the oscillation in the overlap region performs as sinusoidal evolution with a rapid decreased frequency. When the pulses continually propagate from $2 L_{D}$ to $6 L_{D}$, sinusoidal fit oscillation evolves into asymptotic dark solitons oscillation, and the oscillating frequency decreases slowly, resulting in a stable pulses transmission. The decrease rate of the sinusoidal frequency is about 6.5 times than that of asymptotic dark solitons oscillation frequency, a feature clearly seen in the fitting curve slopes of Fig.4.

This can be interpreted by considering the relationship between dispersion length $L_{D}$ and nonlinearity length $L_{N L}$. We can obtain the two parameters from the initial conditions:

$$
\begin{gathered}
L_{D}=\frac{T_{0}^{2}}{\left|\beta_{2}\right|}=\frac{T_{F W H M}^{2}}{4 \ln 2\left|\beta_{2}\right|}=277.4 m \\
L_{N L}=\frac{1}{\gamma P_{0}}=\frac{\sqrt{\pi} T_{0}}{\gamma E}=5.3 \mathrm{~m}
\end{gathered}
$$

It can then be used to calculate parameter $N$ using

$$
N^{2}=\frac{L_{D}}{L_{N L}}=\frac{\gamma P_{0} T_{0}^{2}}{\left|\beta_{2}\right|}=\frac{T_{\text {FWH }}^{2}}{4 \ln 2\left|\beta_{2}\right|}=5.17>1
$$

When the fiber length $L$ is such that $L<L_{D}$ but $L \approx L_{N L}$, pulse evolution in the fiber is governed by SPM that leads to spectral broadening of the pulses. When the fiber length is longer than both $L_{D}$ and $L_{N L}$, dispersion and nonlinearity act together as the pulse propagates along the fiber. The interplay of the GVD and SPM effects can lead to a qualitatively different behavior compared with that expected from GVD or SPM alone. In this condition, both dispersive and nonlinear effects are important to pulse evolution in optical fibers, and the GVD effect slowdown the spectral broadening of the pulses. When the pulses' propagation length is more than $6 L_{D}$, the asymptotic dark 
solitons oscillation disappears and the oscillation becomes irregular.

In order to analyze the relationship between oscillation region and self-similar propagation region, we further study self-similar pulses oscillation characteristics in the sinusoidal fit oscillation region and the asymptotic dark solitons oscillation region.

\section{1 oscillation characteristics in the sinusoidal fit oscillation region}

We investigate the oscillation characteristics of self-similar pulses in the sinusoidal fit oscillation region. From the simulation, sinusoidal oscillation occurs before the pulses enter the self-similar region. Fig. 5 presents the center part of the intensity profiles of a pair of pulses at different fiber length.

At the first stage, when $L<L_{D}$, the amplitude of the interacting pulses increases due to the SPM effect. At the second stage, when $L \geq L_{D}$, the amplitude of the pulses remains constant due to the interplay of the GVD and SPM effects. Meanwhile, the oscillation frequency decreases all the time.

As Fig. 5 shown, when $z$ is equal to $5 / 8 L_{D}$, the pulses begin to interact and create slight sinusoidal oscillation, which has a frequency of $f=62 / \pi=9.873 \mathrm{THz}$ and an oscillation amplitude of $P= \pm 0.5 \mathrm{~W}$ at $P=1 \mathrm{~W}$. When $z=6 / 8 L_{D}$, the sinusoidal oscillation becomes obviously, which amplitude increases to $3.5 \mathrm{~W}$ as the oscillation frequency decreases to $f=9.554 \mathrm{THz}$, and the oscillation amplitude at the edge is much smaller than that at the center. When the propagate distance is $z=9 / 8 L_{D}$, the sinusoidal oscillation tends to be stable with an oscillation amplitude $P=9 \mathrm{~W}$ and a decreased frequency $f=7.325 \mathrm{THz}$. The overlap region increases with the propagate length, and the oscillation amplitude at the edge is almost the same with that at the 
center, which means the whole overlap region presents sinusoidal oscillation with the same frequency and amplitude, as Fig.5 shown. As the pulses continue to propagate, the oscillation amplitude remains unchanged, but the frequency decreases gradually, which reduces to $f=4.777 T H z$ when $z=2 L_{D}$. Fig.5 also shows that the oscillation amplitude changes from sinusoidal fit to asymptotic dark solitons fit although the oscillation frequency still remains sinusoidal fit.

From the analyzation of the self-similar propagation region, the self-similar coefficient $F$ value varies from $40.27 \%$ to $7.99 \%$ when $z$ varies from $5 / 8 L_{D}$ to $2 L_{D}$. This suggests that sinusoidal fit oscillation begins when the pulse has not entered the self-similar propagation region, and almost ends when it has just entered the self-similar propagation region.

\section{2 oscillation characteristics in the asymptotic dark solitons oscillation region}

From table 1, when the overlapping pulses further propagate from $z=2 L_{D}$ to $z=6 L_{D}$, they evolve into a train of asymptotic dark solitons with intensity profile $P(T)=A^{2}\left(1-\sec h^{2}(c * t)\right)$, where $A^{2}$ is the intensity depth of the hole, whose valve is equal to the difference between the maximum and minimum values of pulses' energy. $c$ is a coefficient determining the wave width of the hole (Dianov et al.1989). From Fig.6, as the propagation length increases, the intensity depth decreases while the period of asymptotic dark solitons increases. What is more, the pulses between the adjacent asymptotic dark solitons no longer remain linear propagation, but a slight oscillation. Fig.7 shows the dark soliton fitting curves (solid line) and the overlap 
pulses oscillation envelope (dotted line) relatively.

From Fig.7, the coefficient $c$ remains constant while the intensity depth decreases. Meanwhile, the distance between the adjacent asymptotic dark solitons increase, indicating decreasing of the frequency of asymptotic dark solitons.

The relationship between intensity depth of the hole and propagation length can be seen by referring to Fig.8. When the asymptotic dark solitons propagate from $z=2 L_{D}$ to $z=4 L_{D}$, the decrease rate is faster than that when the asymptotic dark solitons propagate from $z=4 L_{D}$ to $z=6 L_{D}$. When the pulses continue to propagate, the asymptotic dark solitons oscillation disappears and the oscillation become irregular. From the analyzation of the self-similar propagation region, the self-similar coefficient $F$ value varies from $7.99 \%$ to $5.32 \%$ when $z$ varies from $2 L_{D}$ to $6 L_{D}$. This indicates that dark soliton fit oscillations occur within the self-similar pulse propagation region with a small $\mathrm{F}$ value, and ends because of the energy loss due to oscillations.

\section{Summary}

In conclusion, we have analyzed the dynamic evolution of self-similar propagation region for single pulse, sinusoidal fit oscillation region and asymptotic dark soliton oscillation region for a pair of interacting parabolic pulses.

The self-similar propagation region starts at $z=1.8 L_{D}$ and ends at $z=18 L_{D}$ while $F \leq 10 \%$. Self-similar coefficient $F$ and RMS width ratio $K$ have the same evolution trend with fiber length and achieve a minimum value simultaneously when $z=3.5 L_{D}$, indicating optimum output of self-similar pulse. The pulses evolution of the pulses 
interacting region is mainly divided into three parts, which are sinusoidal fit oscillation, asymptotic dark solitons oscillation and irregular oscillation. The decrease rate of the oscillation frequency in the sinusoidal fit oscillation region is about 6.5 times higher than that in the asymptotic dark solitons oscillation region. The sinusoidal fit oscillation region ranges from $5 / 8 L_{D}$ to $2 L_{D}$ while self-similar coefficient $F$ value varies from $40.27 \%$ to $7.99 \%$, which indicates that the sinusoidal fit oscillation region almost occurs before the pulses enter the self-similar propagation region. In the sinusoidal fit oscillation region, the overlap region of self-similar pulses increases with the propagate length, and the whole overlap region finally evolves into sinusoidal oscillation with the same frequency and amplitude. The amplitude of the interacting pulses in the sinusoidal fit oscillation region increases at the first stage and remains constant at the second stage while the oscillation frequency decreases all the time. The dark soliton fit oscillation region ranges from $2 L_{D}$ to $6 L_{D}$ while self-similar coefficient $F$ varies from $7.99 \%$ to $5.32 \%$, which means the dark soliton fit oscillation region occurs within the self-similar pulse propagation region with a smaller $F$. The train of asymptotic dark solitons evolves with a decreased intensity depth and a constant coefficient $c$ while the period of asymptotic dark solitons increases. After that, the asymptotic dark solitons oscillation disappears and the oscillation becomes irregular because of the energy loss due to oscillations.

The results are beneficial in self-similar pulses and high-quality femtosecond pulses generation. It is important to Dense Wavelength Division Multiplexing transmission system which is in heavy demands of light source in wide-range 
wavelength.

\section{Acknowledgements}

This work was supported by National Natural Science Foundation of China (No. 61705045, No. U1601202, No.51675106), Guangdong Provincial Science and Technology Research Project ( No.2015B010104008 , No.2016A030308016 , No.17ZK0091), Foundation of Guangdong Province Science and Technology (No. 2017A090905047), Special and Technology Enterprises of Provincial Science and Technology Enterprises of Small and Medium sized (No. 2016A010119143), Foundation of Guangdong Province Science and Technology (No. 201604010011).

\section{References}

1. Barenblatt, G.I.: Scaling, Self-Similarity, and Intermediate Asymp- totics. Cambridge, UK (1996)

2. Desaix, M.: Wave-breaking-free pulses in nonlinear-optical fibers. J. Opt. Soc. Am. B. 10,1185-1190(1993)

3. Ruehl, A., Prochnow, O., Wandt, D., Krachtet, D., Burgoyne, B., Godbout, N., Lacroix, S.: Dynamics of parabolic pulses in an ultrafast fiber laser. Opt. Lett. 31, 2734-2736(2006)

4. Tamura, K. R., Kubota, T. H., Nakazawa, M.: Fundamentals of stable continuum generation at high repetition rates. IEEE J. Quantum Electron. 36, 773-779(2000)

5. Ozeki, Y., Takushima, Y., Aiso, K., Taira, K., Kikuchi, K.,: Generation of 10GHz similariton pulse trains from $1.2 \mathrm{~km}$-long erbium-doped fibre amplifier for 
application to multi- wavelength pulse sources. Electron. Lett. 40( 2004)

6. Wang, L., Zhu, Y. J., Jiang, D. Y.: Self-similar rogue waves and nonlinear tunneling effects in inhomogeneous nonlinear fiber optics. J. Mod. Opt. 63, 704-714 (2016)

7. Boscolo, S., Latkin, A. I., Turitsyn,S. K.: Passive nonlinear pulse shaping in normally dispersive fiber systems. IEEE J. Quantum Electron. 44, $1196-1203(2008)$

8. Hirooka, T., Nakazawa, M.: Parabolic pulse generation by use of a dispersion-decreasing fiber with normal group-velocity dispersion. Opt. Lett. 29, 498-500(2004)

9. Kibler, B., Billet, C., Lacourt, P. A., Ferriere, R., Dudley, J. M.: All-fiber source of 20 -fs pulses at $1550 \mathrm{~nm}$ using two-stage linear-nonlinear compression of parabolic similaritons. IEEE Photonics Technol. Lett. 18, 1831-1833(2006)

10. Wabnitz, S., Finot,C.: Theory of parabolic pulse propagation in nonlinear dispersion-decreasing optical fiber amplifiers. J. Opt. Soc. Am. B. 25, 614-621(2008)

11. Ghosh, D., Basu, M., Sarkar, S.: Generation of self-similar parabolic pulses by designing normal dispersion decreasing fiber amplifier as well as its staircase substitutes. J. Light. Technol. 27, 3880-3887(2009)

12. Bale, B. G., Boscolo, S.: Impact of third-order fibre dispersion on the evolution of parabolic optical pulses. J. Opt. 12, 015202-015207 (2010)

13. Ghosh, D., Basu, M.: Designing suitable dispersion decreasing active fibers to 
generate parabolic pulses in presence of macrobending. Opt Quant Electron.49:8 (2017)

14. He, J. R., Yi, L.: Exact optical self-similar solutions in a tapered graded-index nonlinear-fiber amplifier with an external source. Opt. Commun. 320, $129-137(2014)$

15. Finot, C., Millot, G.: Interaction between optical parabolic pulses in a Raman fiber amplifier. Opt. Express. 13, 5825-5830(2005)

16. Finot, C., Dudley, J. M., Millot, G.: Generation of dark solitons by interaction between similaritons in Raman fiber amplifiers. Opt. Fiber Technol. 12, 217-226(2006)

17. Liu, Y. L., Luo, A. P., Luo, Z. C., Xu, W. C.: Suppression of parabolic pulse-pair interaction using dispersion-managed fiber links with non-zero dispersion. J. Mod. Opt. 58, 1004-1011(2011)

18. Ponomarenko, S. A., Agrawal, G. P.: Nonlinear interaction of two or more similaritons in loss- and dispersion-managed fiber. J. Opt. Soc. Am. B. 25, 983-989(2008)

19. Loomba, S., Mani Rajan, M. S., Gupta, R., Kaur, H., Kumar, C. N.: Nonlinear tunneling of optical similaritons in a tapered graded-index nonlinear waveguide. Opt. Commun. 324, 286-295(2014)

20. Kumar De, K., Goyal, A., Raju, T. S., Kumar, C. N., Panigrahi, P. K: Riccati parameterized self-similar waves in two-dimensional graded-index waveguide. Opt. Commun. 341, 15-21(2015) 
21. Ghosh, D., Chowdhury, D., Basu, M.: Silica based highly nonlinear fibers to generate parabolic self-similar pulses. Opt. Quantum Electron. 47, 2615-2635(2015)

22. Krishna Ghosh, B., Ghosh, D., Basu, M.: Prospective use of a normally dispersive step-index chalcogenide fiber in nonlinear pulse reshaping. Appl. Opt. $57,3348-3356(2018)$

23. Zhang Q., Gao J.: Generation of excellent self-similar pulses in a dispersion-decreasing fiber. Optik. 122, 1753-1756(2011)

24. Silva da M.G., Nobrega K.Z., Sombra A.S.B.: Analysis of soliton switching in dispersion-decreasing fiber couplers, Optics Communications. 171,351-364 (1999)

25. Amir M., Hamid H.H.: Optimum Dispersion Profile for Compression of Fundamental Solitons in Dispersion Decreasing Fibers, IEEE Journal of Quantum Electronics. 33, 620-628(1997)

26. Zhang Q., Li H., Wu L., Gao J: Influence of dispersion distribution on the propagation and compression of self-similar optical beam, Eur. Phys. J. D 73: 26(2019)

27. Dianov, E. M., Mamyshev, P. V., Prokhorov, A. M., Chernikov, S. V.: Generation of a train of fundamental solitons at a high repetition rate in optical fibers. OPTICS LETTERS. 18, 1008-1010(1989) 



\section{Figures}

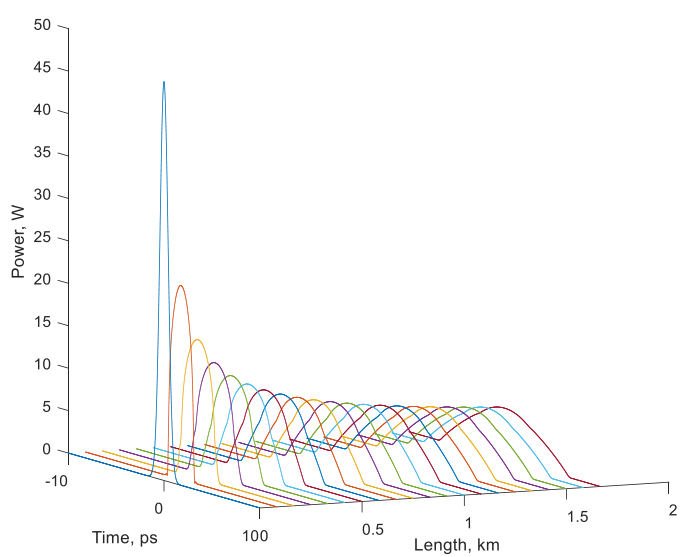

Fig.1 Single pulse evolution in the ND-DDF from $\mathrm{z}=0$ to $\mathrm{z}=6 \mathrm{~L}_{\mathrm{D}}$

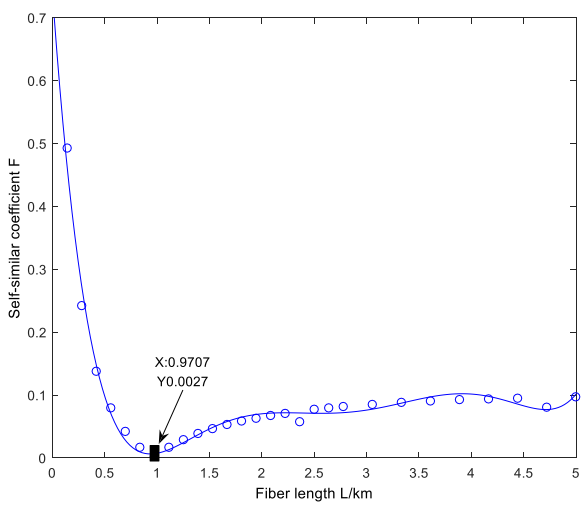

(a)

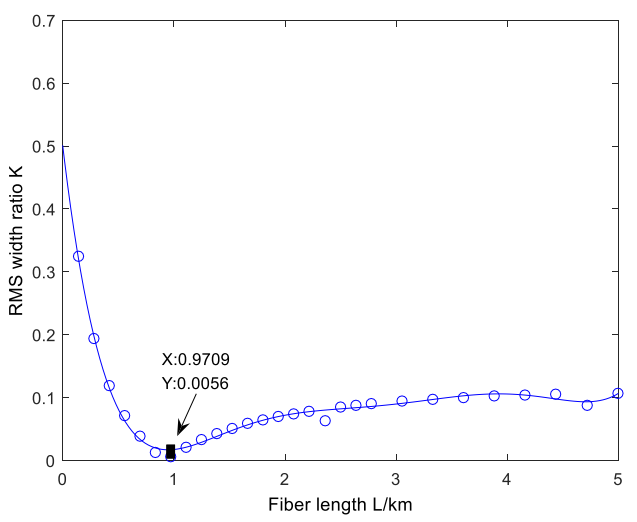

(b)

Fig.2 (a) Relationship between self-similar coefficient F and fiber length L; (b) Relationship between RMS width ratio $\mathrm{K}$ and fiber length $\mathrm{L}$.

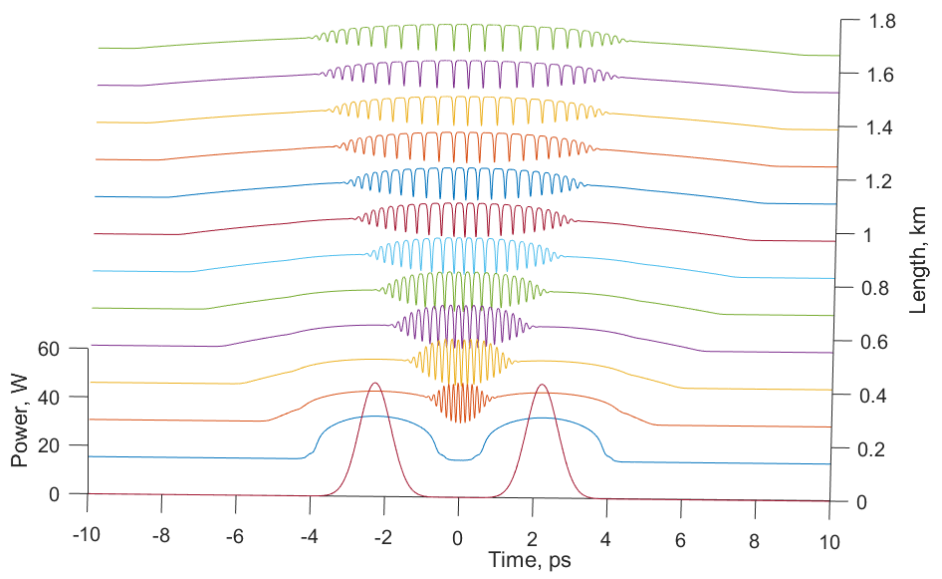

Fig.3 Waveform evolution of self-similar pulses in DDF 


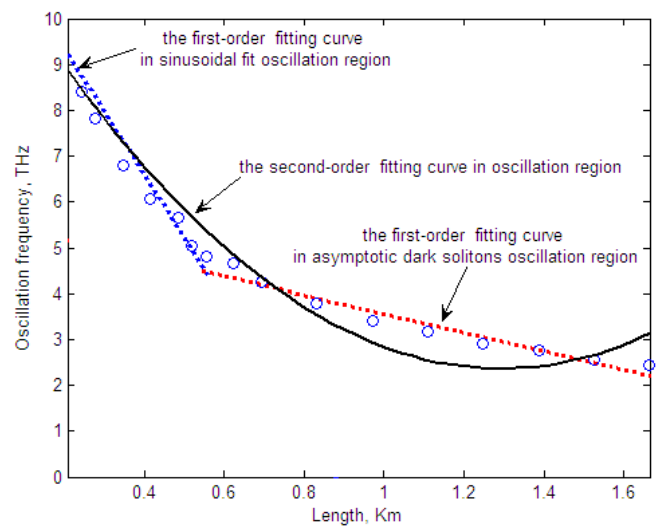

Fig. 4 Relationship between the oscillation frequency and propagation length
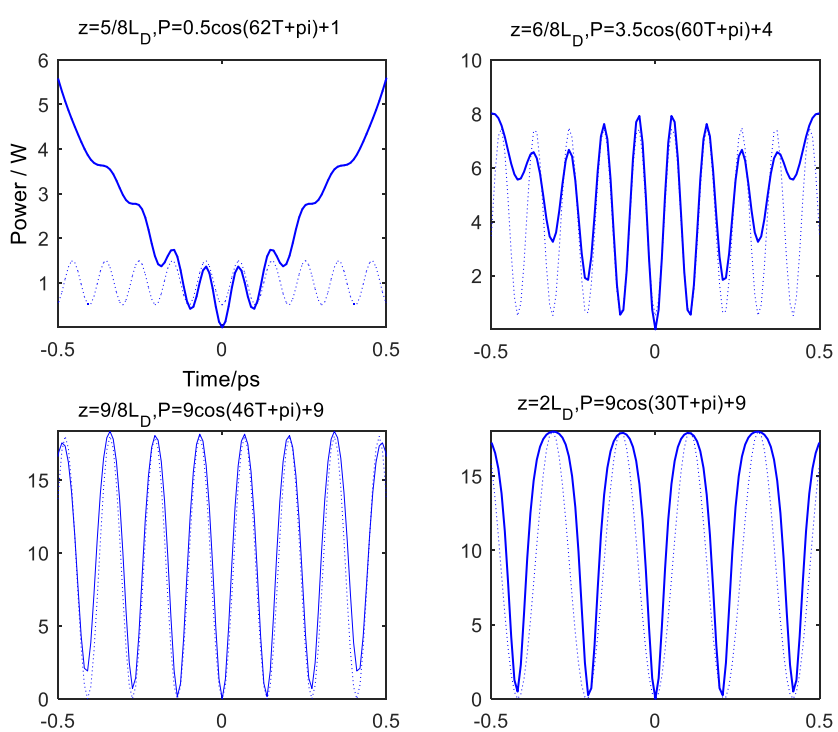

Fig.5 Center part of the intensity profiles of a pair of pulses at different fiber length in the sinusoidal oscillation region: simulations (solid line) and sinusoidal fit (dotted line)

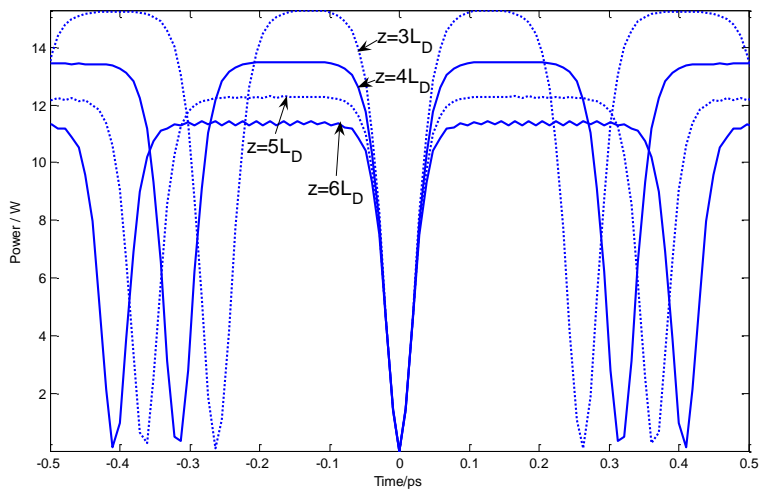

Fig.6 Center part of the intensity profiles of a pair of pulses at different fiber length in the asymptotic dark solitons oscillation region 

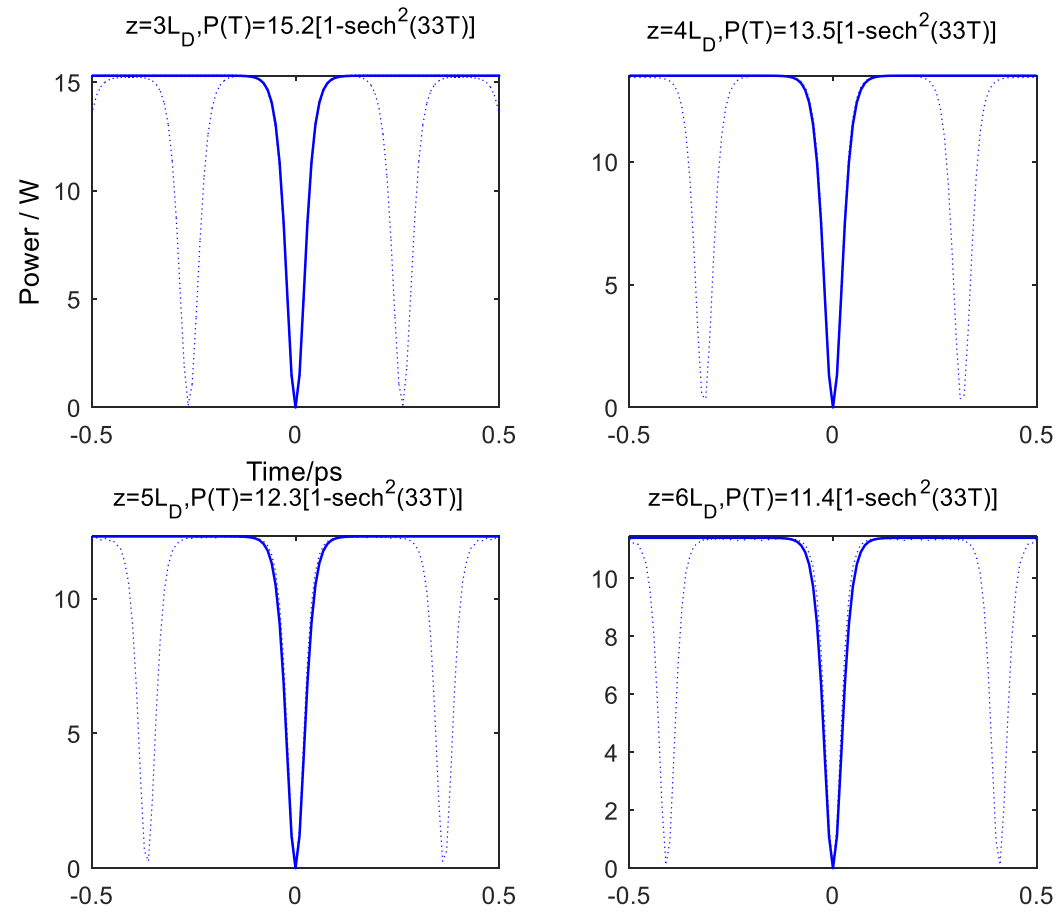

Fig.7 Center part of the intensity profiles of a pair of pulses at different fiber length in the asymptotic dark solitons oscillation region: simulations (dotted line) and asymptotic dark solitons fit (solid line)

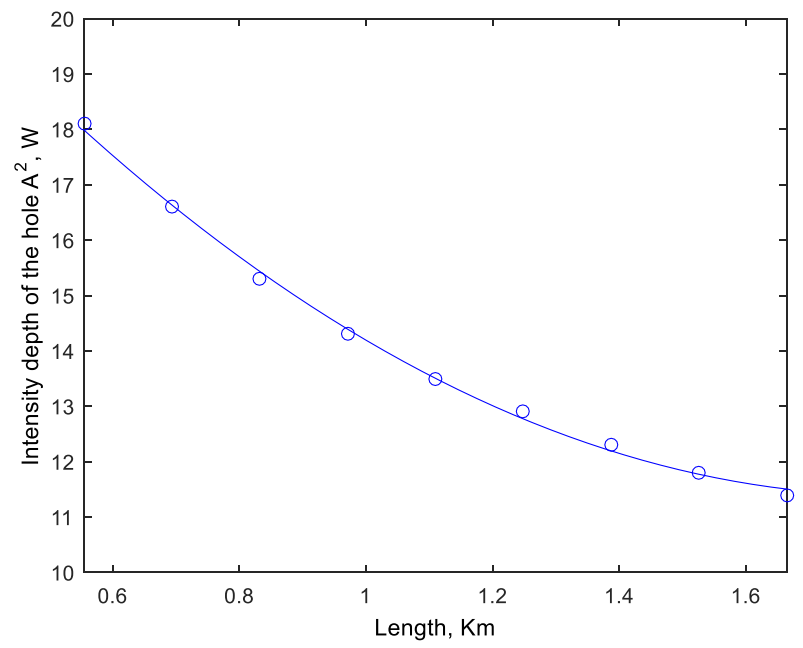

Fig.8 Relationship between intensity depth of the hole $\mathrm{A}^{2}$ and propagation length 


\section{Tables}

Table 1. Relationship between the oscillation period and fiber length

\begin{tabular}{|c|c|c|c|c|c|}
\hline & $\begin{array}{l}\text { Length } \\
\text { (z/Km) }\end{array}$ & $\begin{array}{c}\text { Oscillation } \\
\text { period (T/ps) }\end{array}$ & & $\begin{array}{l}\text { Length } \\
(\mathrm{z} / \mathrm{Km})\end{array}$ & $\begin{array}{c}\text { Oscillation } \\
\text { period }(\mathrm{T} / \mathrm{ps})\end{array}$ \\
\hline \multirow[b]{4}{*}{ Sinusoidal fit } & $5 / 8 \mathrm{~L}_{\mathrm{D}}$ & 0.093 & \multirow{9}{*}{$\begin{array}{l}\text { Asymptotic dark } \\
\text { solitons oscillation }\end{array}$} & $2 \mathrm{~L}_{\mathrm{D}}$ & 0.208 \\
\hline & $6 / 8 \mathrm{~L}_{\mathrm{D}}$ & 0.109 & & $2.5 \mathrm{~L}_{\mathrm{D}}$ & 0.235 \\
\hline & $7 / 8 \mathrm{~L}_{\mathrm{D}}$ & 0.119 & & $3 \mathrm{~L}_{\mathrm{D}}$ & 0.265 \\
\hline & $8 / 8 \mathrm{~L}_{\mathrm{D}}$ & 0.128 & & $3.5 \mathrm{~L}_{\mathrm{D}}$ & 0.294 \\
\hline oscillation & $10 / 8 \mathrm{~L}_{\mathrm{D}}$ & 0.147 & & $4 \mathrm{LD}_{\mathrm{D}}$ & 0.315 \\
\hline \multirow[t]{4}{*}{ region } & $12 / 8 \mathrm{~L}_{\mathrm{D}}$ & 0.165 & & $4.5 \mathrm{~L}_{\mathrm{D}}$ & 0.343 \\
\hline & $14 / 8 \mathrm{~L}_{\mathrm{D}}$ & 0.177 & & $5 \mathrm{~L}_{\mathrm{D}}$ & 0.363 \\
\hline & $15 / 8 \mathrm{~L}_{\mathrm{D}}$ & 0.198 & & $5.5 \mathrm{~L}_{\mathrm{D}}$ & 0.391 \\
\hline & $2 \mathrm{~L}_{\mathrm{D}}$ & 0.208 & & $6 \mathrm{~L}_{\mathrm{D}}$ & 0.412 \\
\hline
\end{tabular}




\section{Figures}

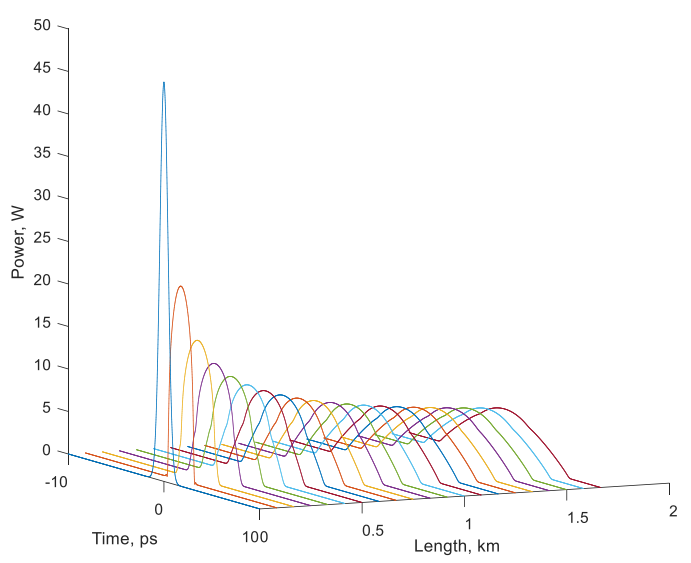

Fig.1 Single pulse evolution in the ND-DDF from $\mathrm{z}=0$ to $\mathrm{z}=6 \mathrm{~L}_{\mathrm{D}}$

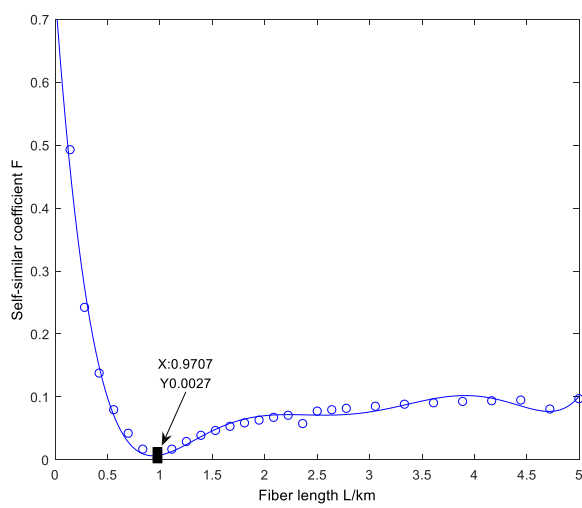

(a)

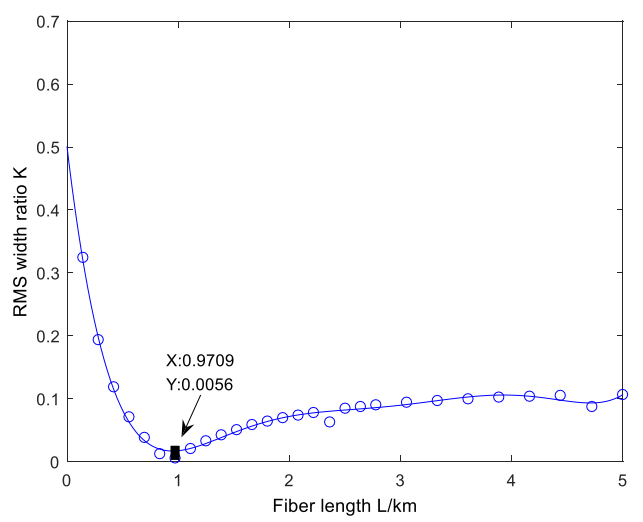

(b)

Fig.2 (a) Relationship between self-similar coefficient F and fiber length L; (b) Relationship between RMS width ratio $\mathrm{K}$ and fiber length $\mathrm{L}$.

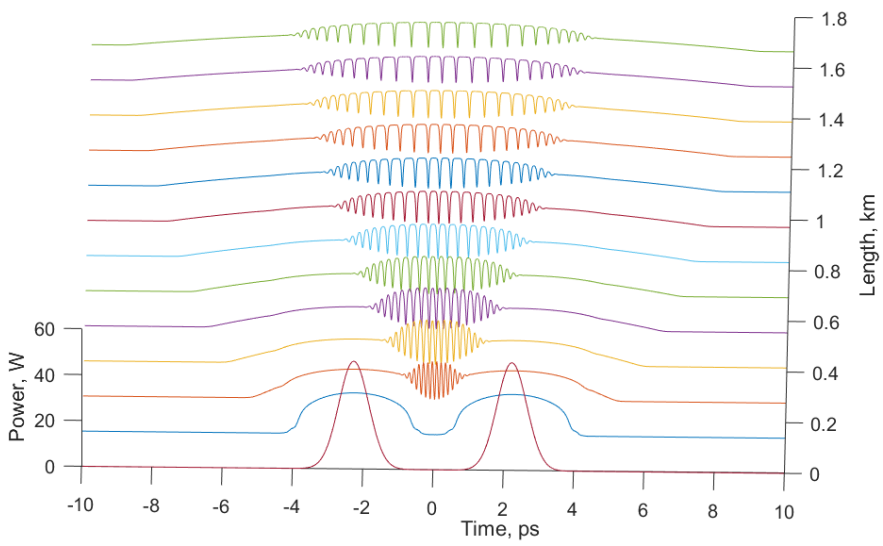

Fig.3 Waveform evolution of self-similar pulses in DDF 


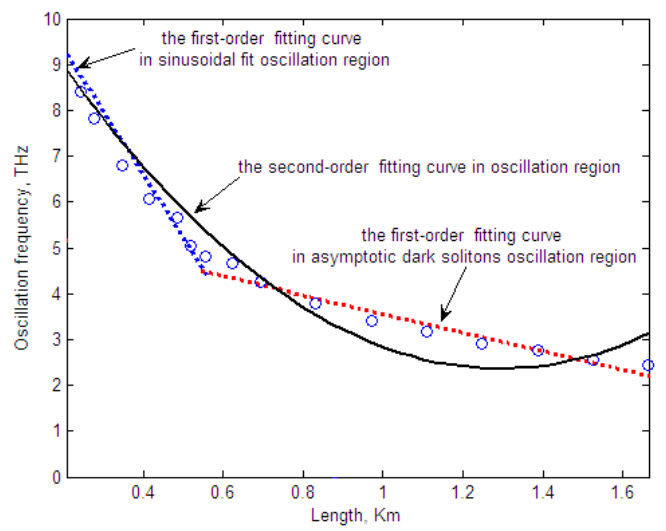

Fig. 4 Relationship between the oscillation frequency and propagation length
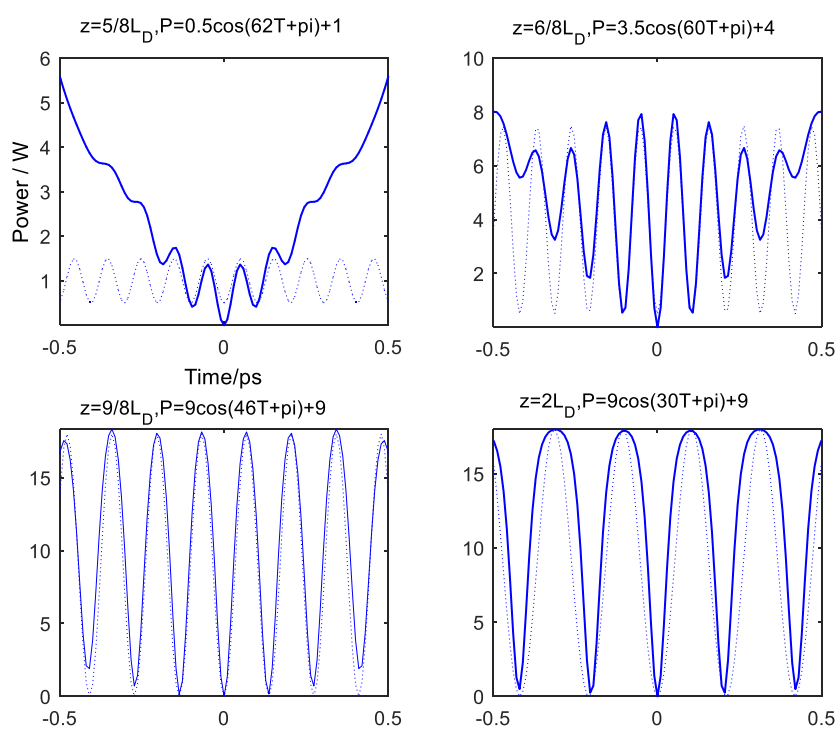

Fig.5 Center part of the intensity profiles of a pair of pulses at different fiber length in the sinusoidal oscillation region: simulations (solid line) and sinusoidal fit (dotted line)

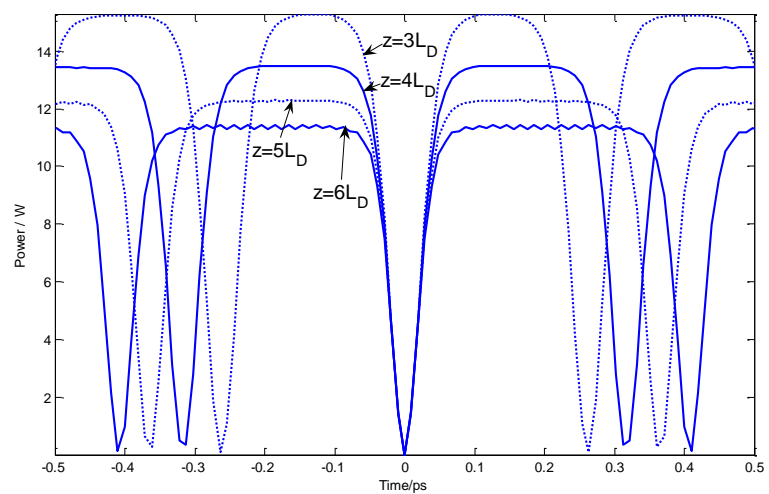

Fig.6 Center part of the intensity profiles of a pair of pulses at different fiber length in the asymptotic dark solitons oscillation region 

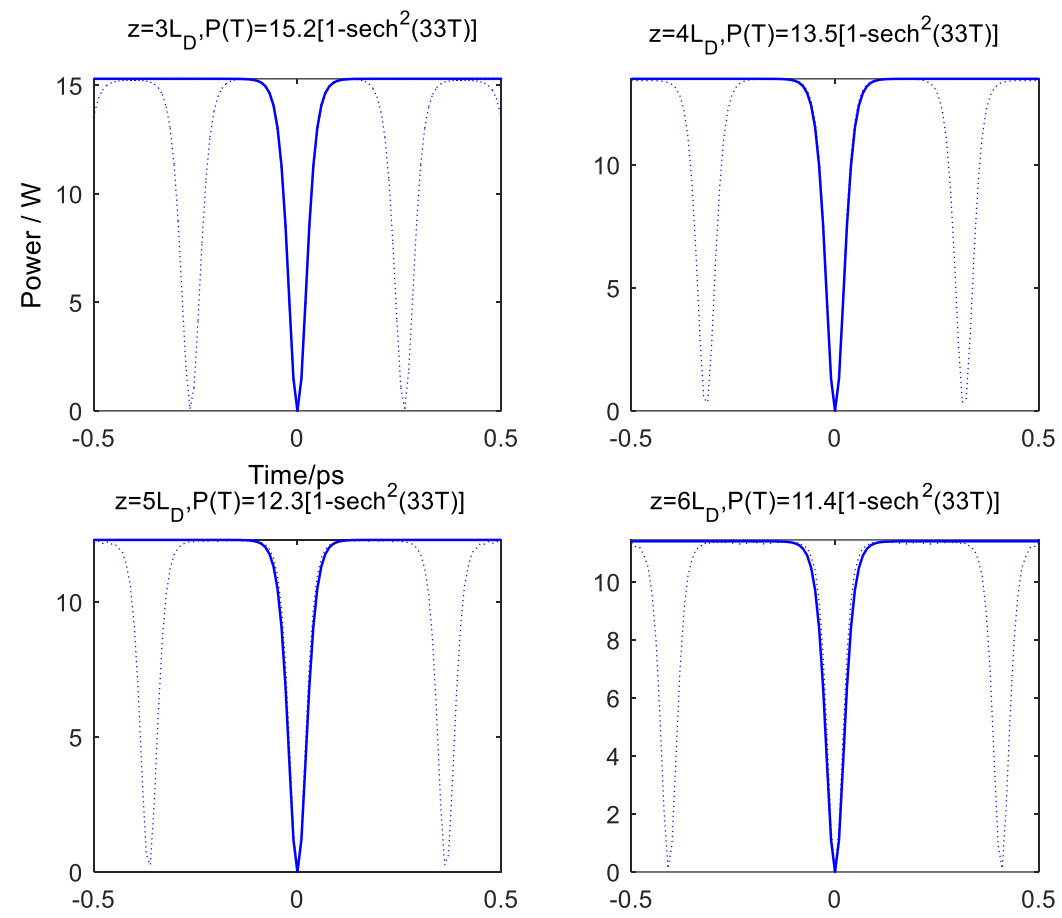

Fig.7 Center part of the intensity profiles of a pair of pulses at different fiber length in the asymptotic dark solitons oscillation region: simulations (dotted line) and asymptotic dark solitons fit (solid line)

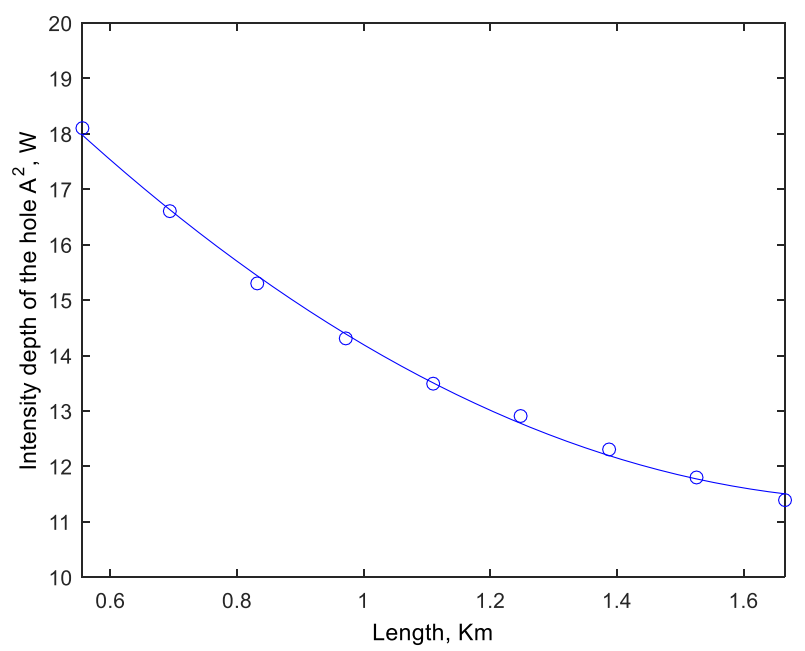

Fig.8 Relationship between intensity depth of the hole $\mathrm{A}^{2}$ and propagation length 


\section{Tables}

Table 1. Relationship between the oscillation period and fiber length

\begin{tabular}{|c|c|c|c|c|c|}
\hline & $\begin{array}{l}\text { Length } \\
(\mathrm{z} / \mathrm{Km})\end{array}$ & $\begin{array}{c}\text { Oscillation } \\
\text { period }(\mathrm{T} / \mathrm{ps})\end{array}$ & & $\begin{array}{l}\text { Length } \\
(\mathrm{z} / \mathrm{Km})\end{array}$ & $\begin{array}{l}\text { Oscillation } \\
\text { period }(\mathrm{T} / \mathrm{ps})\end{array}$ \\
\hline \multirow[b]{4}{*}{ Sinusoidal fit } & $5 / 8 \mathrm{~L}_{\mathrm{D}}$ & 0.093 & \multirow{9}{*}{ Asymptotic dark } & $2 \mathrm{~L}_{\mathrm{D}}$ & 0.208 \\
\hline & $6 / 8 \mathrm{~L}_{\mathrm{D}}$ & 0.109 & & $2.5 \mathrm{~L}_{\mathrm{D}}$ & 0.235 \\
\hline & $7 / 8 \mathrm{LD}_{\mathrm{D}}$ & 0.119 & & $3 \mathrm{~L}_{\mathrm{D}}$ & 0.265 \\
\hline & $8 / 8 \mathrm{LD}_{\mathrm{D}}$ & 0.128 & & $3.5 \mathrm{LD}_{\mathrm{D}}$ & 0.294 \\
\hline \multirow{5}{*}{$\begin{array}{l}\text { oscillation } \\
\text { region }\end{array}$} & $10 / 8 \mathrm{~L}_{\mathrm{D}}$ & 0.147 & & $4 \mathrm{~L}_{\mathrm{D}}$ & 0.315 \\
\hline & $12 / 8 \mathrm{~L}_{\mathrm{D}}$ & 0.165 & & $4.5 \mathrm{~L}_{\mathrm{D}}$ & 0.343 \\
\hline & $14 / 8 \mathrm{~L}_{\mathrm{D}}$ & 0.177 & & $5 \mathrm{~L}_{\mathrm{D}}$ & 0.363 \\
\hline & $15 / 8 \mathrm{~L}_{\mathrm{D}}$ & 0.198 & & $5.5 \mathrm{LD}_{\mathrm{D}}$ & 0.391 \\
\hline & $2 \mathrm{~L}_{\mathrm{D}}$ & 0.208 & & $6 \mathrm{~L}_{\mathrm{D}}$ & 0.412 \\
\hline
\end{tabular}

